\title{
A prática da terapia ocupacional junto ao idoso com alterações ortopédicas em um hospital de urgência e emergência
}

\section{Occupational therapy practice to the elderly with orthopedic condition in an emergency hospital}

\author{
Helena Auler Loureiro ${ }^{1}$, Kênia Lara Silva ${ }^{2}$, Marcela Aline Fernandes Braga ${ }^{3}$ \\ http://dx.doi.org/10.11606/issn.2238-6149.v30i1p53-61
}

Loureiro HA, Silva KL, Braga MAF. A prática da terapia ocupacional junto ao idoso com alterações ortopédicas em um hospital de urgência e emergência. Rev Ter Ocup Univ São Paulo. 2019 jan.-abr:;30(1):53-61.

RESUMO: Objetivo: Analisar a proposta de implementação do serviço de terapia ocupacional, considerando as avaliações e as intervenções da Terapia Ocupacional (TO) junto ao idoso com alterações ortopédicas, em um hospital de urgência e emergência. Métodos: Estudo longitudinal retrospectivo e descritivo, com 125 idosos internados em um hospital de urgência e emergência em Belo Horizonte, por alterações ortopédicas, atendidos pela TO da equipe de Ortopedia, internados após a estruturação do serviço de TO na Ortopedia, de março de 2015 a abril de 2016. Foram coletadas nos prontuários: informações sociodemográficas, clinicas, funcionais e procedimentos específicos da TO. Foi realizada a análise descritiva do material. Resultados: Foi encontrada maior incidência de fratura no gênero feminino. A fratura de fêmur apresentou maior frequência, seguida das fraturas de membro superior. As principais intervenções realizadas pela TO foram intervenção educativa, intervenção nas funções mentais, intervenção adaptativa, intervenção restauradora da atividade de mudança de posição básica do corpo. A revisão de prótese foi o procedimento ortopédico de maior média de atendimentos de TO. Conclusões: A TO dirige suas intervenções na prevenção e/ ou tratamento de possíveis complicações, que podem aumentar o tempo de permanência hospitalar e gerar maior declínio funcional para o idoso, reduzindo sua funcionalidade em contexto domiciliar.

DESCRITORES: Idoso; Terapia ocupacional; Terapia ocupacional/ normas; Ortopedia.
Loureiro HA, Silva KL, Braga MAF. Occupational therapy practice to the elderly with orthopedic condition in an emergency hospital. Rev Ter Ocup Univ São Paulo. 2019 Jan.-Apr.;30(1):53-61.

ABSTRACT: Objective: To analyze the Occupational Therapy (OT) practice with the orthopedic alterations elderly in an urgency and emergency hospital. Methods: Retrospective longitudinal study with 125 hospitalized due to orthopedic alterations aged patients in an urgency and emergency hospital in Belo Horizonte, attended by the OT of the Orthopedic team, hospitalized after the structuring of the OT in Orthopedics service, from March 2015 to April 2016. The sociodemographic, clinical, functional and specifics procedures of OT. The descriptive analysis of the material was carried out. Results: A higher incidence of fracture was found in females. The femoral fracture had a higher frequency, followed by fractures of the upper limb. The main interventions performed by OT were educational intervention, intervention in mental functions, adaptive intervention and restorative intervention of the activity basic body position change. The hip revision was the orthopedic procedure with the highest average number of OT visits. Conclusions: The OT focuses its interventions with this population on the prevention and/or treatment of possible complications, which can increase hospital stay time and generate greater functional decline for the elderly, reducing their functionality in home environment.

KEYWORDS: Aged; Occupational therapy; Occupational therapy/ standards; Orthopedics.

Trabalho de Conclusão de Curso junto ao programa de Residência na Saúde do Idoso do Hospital Risoleta Tolentino Neves de Belo Horizonte, MG.

1. Terapeuta Ocupacional. Residência na Saúde do Idoso do Hospital Risoleta Tolentino Neves, Belo Horizonte, MG, BR. OCID: https://orcid.org/ 0000-0001-8991-8878. Email: helena.auler8@gmail.com

2. Enfermeira. Doutora em Enfermagem. Docente junto à Graduação e Pós-Graduação em Enfermagem da Universidade Federal de Minas Gerais, Belo Horizonte, MG, BR. OCID: https://orcid.org/0000-0003-3924-2122. Email: kenialara17@gmail.com

3. Terapeuta Ocupacional. Mestre em Neurociências pela Universidade Federal de Minas Gerais, Belo Horizonte, MG, BR. Preceptora da Residência em Saúde do Idoso do Hospital Risoleta Tolentino Neves. OCID: http://orcid.org/0000-0002-3364-8087. Email: marcelabraga.ot@gmail.com

Endereço para correspondência: Rua Itaí, 923 apt. 302. Santa Efigênia, Belo Horizonte, MG. CEP 30260290 


\section{INTRODUÇÃO}

$\mathrm{O}$ envelhecimento populacional tem sido considerado nas últimas décadas um fenômeno de grande relevância, que ocorre em todo o mundo, com características distintas nos países em desenvolvimento ${ }^{1,2}$. No Brasil, entre 1997 e 2007, a população de idosos de 60 a 69 anos aumentou $21,6 \%$, e a de mais de 80 cresceu $47,8 \%{ }^{3}$. Uma população em processo de envelhecimento tem como consequência o aumento importante e progressivo dos níveis de morbidade e aumento de comorbidade associadas a processos crônicos, degenerativos e, na maioria das vezes, incapacitantes, como alterações no equilíbrio, visão, marcha, cognição entre outros ${ }^{1,2,4}$.

Essas limitações associadas a condições agudas ou crônicas tornam os idosos mais suscetíveis aos riscos ambientais, levando muitas vezes à ocorrência de quedas ${ }^{5}$. Tais quedas têm se apresentado como o maior fator precursor de fraturas em idosos, potente desencadeador de consequências, capazes de serem prejudiciais à qualidade de vida deste idoso, gerando imobilidade e dependência ${ }^{1,4,5,6}$.

Atualmente as quedas e, consequentemente, as fraturas no idoso representam um problema de saúde pública, não só devido à sua alta prevalência, mas também à implicação nos níveis de morbidade e mortalidade, além da sua extensão como um problema social ${ }^{1,4,5,7,8,9,10,11}$. A hospitalização, muito frequentemente nesses casos, diminui a capacidade funcional, principalmente quando ocorre alguma intervenção cirúrgica gerando mudanças na qualidade de vida do idoso e sua família, muitas vezes, irreversíveis ${ }^{6,8,9,10}$. A atuação da equipe multiprofissional junto a este idoso em contexto hospitalar deve ocorrer de forma breve e eficaz, prevenindo complicações secundárias à internação, uma vez que com poucos dias de hospitalização o idoso pode apresentar declínio funcional, levando à dependência parcial ou completa para a realização das Atividades de Vida Diária (AVDs) $)^{6,9,11,12,13}$.

As alterações ortopédicas devido a fraturas afetam o desempenho ocupacional, restringindo a participação nas atividades cotidianas, por impactarem funções e estruturas do corpo necessárias para a execução de tarefas ${ }^{11,15}$. É sabido que a Terapia Ocupacional deve intervir na capacitação das pessoas que apresentam algum declínio funcional, prevenindo complicações e possibilitando através de diversas intervenções focadas na funcionalidade, que elas voltem a desempenhar tarefas que compõem seu cotidiano, permitindo a plena execução de seus papéis sociais em seus contextos de vida ${ }^{11,15,16,17}$.

No Hospital Risoleta Tolentino Neves, a equipe responsável pelo acompanhamento do idoso com alterações ortopédicas conta com as seguintes especialidades: geriatria, ortopedia, fonoaudiologia, farmácia, nutrição, enfermagem e terapeuta ocupacional. Além desses profissionais, há a possibilidade de acompanhamento por parte de outras especialidades como, fisioterapia, psicologia, assistente social, mediante solicitação de interconsulta.

O setor de Terapia Ocupacional (TO) no Hospital Risoleta Tolentino Neves passou por um processo de estruturação do seu serviço na equipe da Ortopedia no ano de 2014. A partir desta data a equipe de Ortopedia passou a contar com um profissional de Terapia Ocupacional na equipe, e um residente de TO na Saúde do Idoso. Com a estruturação do serviço, foi elaborado um documento de orientação de condutas com o objetivo de padronizar e organizar as principais avaliações e intervenções da TO na ortopedia. Este documento teve como eixo norteador a Classificação Internacional de Incapacidade, Funcionalidade e Saúde (CIF) proposta pela Organização Mundial de Saúde (OMS) como nova compreensão do processo saúde e doença, trazendo conceitos renovadores que são centrais neste processo ${ }^{14}$.

O presente trabalho tem como objetivo descrever e analisar as intervenções bem como a frequência de atendimento da Terapia Ocupacional junto ao idoso com alterações ortopédicas, em um hospital de urgência e emergência, considerando um possível exemplo de raciocínio para o serviço de terapia ocupacional com intervenções destinadas ao idoso para realização de investigações que indiquem os avanços e desafios neste processo.

Há uma lacuna na literatura nacional acerca das avaliações e intervenções da terapia ocupacional com esta população internada. Apesar do impacto significativo dessas ocorrências na prática atual da terapia ocupacional, a área não tem dedicado especial atenção ao problema, o que se configura como questão a ser sanada. $\mathrm{O}$ enfoque aqui trabalhado visa, além de promover a reflexão a este respeito, contribuir para a ampliação do alcance do terapeuta ocupacional em seu campo de trabalho.

\section{METODOLOGIA}

Trata-se de um estudo longitudinal retrospectivo e descritivo, realizado no Hospital Universitário Risoleta Tolentino Neves (HRTN) da Universidade Federal de Minas Gerais (UFMG) em Belo Horizonte, MG, no período de março de 2015 a abril de 2016 com pacientes idosos internados na enfermaria por alterações Ortopédicas acompanhados pela equipe de Terapia Ocupacional.

A coleta dos dados foi feita a partir dos registros de prontuários da Terapia Ocupacional e da Ortopedia. Nos prontuários da Terapia Ocupacional foram coletadas informações sociodemográficas, informações sobre 
avaliações e intervenções específicas realizadas pela TO. Já do prontuário da Ortopedia foram coletadas informações sobre o motivo da lesão, qual tipo de alteração ortopédica o idoso apresentava, se foi realizada alguma intervenção cirúrgica e qual tipo de intervenção foi feita.

Foram incluídos no estudo idosos, com idade igual ou superior a 60 , com alterações ortopédicas, internados na enfermaria, atendidos pelo menos uma vez pela Terapia Ocupacional da Ortopedia, internados após a estruturação do serviço de Terapia Ocupacional na Ortopedia.

Foram excluídos os atendimentos desses pacientes realizados pela Terapia Ocupacional quando o profissional não estava integrado à equipe da Ortopedia. Isso ocorre quando há a transferência do cuidado para outro setor do hospital, como por exemplo CTI ou Pronto Atendimento.

Foi feita a análise retrospectiva dos prontuários de 125 pacientes que respondiam aos critérios de inclusão. Foi realizada a análise descritiva do material, extraindo as frequências relativas a cada variável e foram feitas análises das médias e das proporções dos dados. Foi analisada a distribuição dos dados para verificar normalidade pelas bibliotecas básicas do R versão 3.3.1.

\section{Estruturação do Serviço de Terapia Ocupacional na equipe de Ortopedia}

O Serviço de Terapia Ocupacional do HRTN na Ortopedia conta sempre com um profissional fixo e um residente de Terapia Ocupacional na Saúde do Idoso, que permanece no campo por três meses. A seleção da amostra se deu por relatório gerado pelo prontuário eletrônico de pacientes internados no período determinado e acessado pela equipe por passômetro médico, um sistema de controle interno do cenário do qual a TO tem acesso para verificar quais serão os pacientes elegíveis para acompanhamento. Portanto, através do passômetro eletrônico, o TO é capaz de saber quais são os pacientes internados por questões ortopédicas e organizar o serviço de assistência feito por ele e pelo residente. Todos os pacientes do passômetro da Ortopedia são acompanhamento pela Terapia Ocupacional assim que são internados até a alta hospitalar.

A partir da estruturação do serviço de Terapia Ocupacional na Ortopedia, o critério de seleção dos instrumentos de avaliação teve como referência os domínios da Classificação Internacional de Funcionalidade, Incapacidade e Saúde (CIF). No Hospital Risoleta Tolentino Neves, o processo de avaliação da TO na equipe da Ortopedia, consiste em: histórico ocupacional colhido por meio de entrevista semiestruturada, exame físico para avaliar funções e estruturas do corpo, e aplicação de dois protocolos padronizados (o Mini Exame do Estado Mental MEEM; e a Medida de Independência Funcional - MIF) para avaliação da função do corpo assim como da atividade (Figura 1). A escolha por estes instrumentos se deu após análise da literatura a fim de verificar avaliações padronizadas que fossem validadas e informativas ao profissional.

Para a coleta do histórico ocupacional o Terapeuta Ocupacional aborda o paciente e o cuidador, quando presente, para realizar uma entrevista semiestruturada, com objetivo de traçar o perfil ocupacional que contemple a rotina, nível de participação nas AVD e participação social anterior à fratura. Bem como ter conhecimento dos interesses, valores, crenças e hábitos do idoso. Determinando os papéis ocupacionais e fatores ambientais que estão relacionados ao contexto de vida de cada idoso.

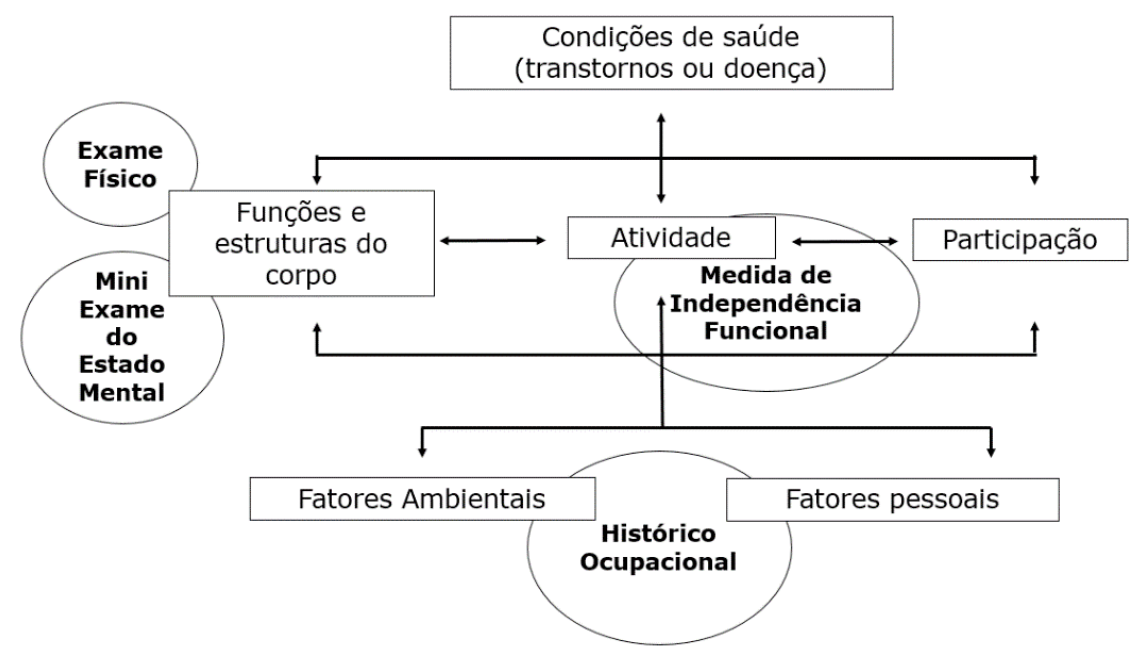

Figura 1 - Avaliação da TO e sua relação com a CIF 
Já o exame físico visa elaborar um diagnóstico para identificar fatores nas funções e estruturas do corpo que interferem ou possam interferir após a evolução do trauma com a independência funcional, tornando-se fatores limitantes de atividade e restringindo a participação. Fazem parte da avaliação relacionada as funções e estruturas do corpo: função sensorial e dor, força muscular, amplitude de movimento das articulações e edema.

Para avaliar a função cognitiva é utilizado o Mini Exame do Estado Mental (MEEM). O MEEM é um teste de rastreio cognitivo, que compreende 11 itens, agrupados em sete categorias, cada uma com o objetivo de avaliar um grupo de funções cognitivas específicas. A pontuação total varia de zero a 30 e possui notas de corte diferenciadas para graus distintos de escolaridade. Os cortes utilizados neste estudo foram: para analfabetos, escore igual a 20; para 1 a 4 anos de escolaridade, escore igual a 25; de 5 a 8 anos de escolaridade, 26; de 9 a 11 anos de escolaridade, 28; e para idosos com escolaridade superior a 11 anos, 29 pontos $^{19}$.

Para avaliar o desempenho deste idoso nas Atividades de Vida Diária (AVDs) durante à internação hospitalar é utilizada a Medida de Independência Funcional (MIF). A MIF é um instrumento validado para uso no Brasil. Ela é composta por 18 itens que avaliam uma série de tarefas motoras e cognitivas de vida diária, entre elas estão o autocuidado, transferências, locomoção, controle esfincteriano, comunicação e cognição social, que inclui memória, interação social e resolução de problemas. Cada atividade recebe uma pontuação de 1 (dependência total) a 7 (independência completa); a pontuação total nestes casos vai variar de 18 a 126, quando menor a pontuação, maior o grau de dependência ${ }^{20}$.

Já em relação as intervenções da Terapia Ocupacional, a forma de documentação no prontuário eletrônico teve como base de raciocínio clínico a $\mathrm{CIF}^{16}$. Utiliza-se a seguinte nomenclatura para a descrição das intervenções da TO no prontuário eletrônico dos pacientes: 1) Intervenção restauradora ou da manutenção da função do corpo - todas as intervenções focadas na restauração de funções do corpo, funções fisiológicas dos sistemas do corpo - que tem como subcategorias as funções mental, neuromusculoesquelética, protetora e reparadora da pele e sensorial. São exemplos destas intervenções a estimulação cognitiva dentro das AVD, a mobilização passiva, ativa ou ativo assistida, massagem retrograda para diminuição de edema, dentre outros; 2) Intervenção restauradora ou da manutenção da atividade de mobilidade que tem como subcategoria mudar a posição básica do corpo, manter a posição básica do corpo e auto transferir-se. São exemplos destas intervenções treino de mudança de decúbito, as transferências da cadeira de banho para o leito, dentre outros; 3) treino funcional, que é o próprio treino das AVD adequando os níveis de exigência da tarefa, treinando estratégias específicas para obter sucesso na atividade e evitar riscos, tem as seguintes atividades como subcategorias que podem ser treinadas junto ao paciente: comer, beber, cuidar dos dentes, cuidar dos cabelos, despir e vestir e cuidar da pele; 4) intervenção educativa, que consiste nas orientações realizadas pelo TO ao paciente e/ou cuidador principal, orientações sobre novas estratégias à serem adotadas para melhor desempenho das tarefas com segurança, ou sobre uso de dispositivos confeccionados como elevador de perna, andador, cadeira de banho, entre outros; e 4) intervenção adaptativa, que consiste na confecção e/ou utilização de dispositivos para compensar funções facilitando o desempenho das AVD, por exemplo, elevador de perna para auxiliar no movimento da perna recém fraturada, escada de cama, para auxiliar na flexão do tronco no momento de mudança de decúbito, dispositivos de posicionamento para aliviar regiões que apresentam risco de lesão por pressão (escaras) ou manter o membro recém fraturado bem posicionado, entre outros.

Como abordado anteriormente, o TO inicia o acompanhamento ao paciente assim que este é internado até o momento da alta hospitalar. Muitos desses pacientes passam por procedimentos cirúrgicos durante à internação. Os que não passam por cirurgia há apenas a imobilização e o tratamento é chamado de tratamento conservador. A depender do tipo de tratamento escolhido pela equipe ortopédica o TO avalia e seleciona as intervenções que devem ser realizadas junto a este paciente ${ }^{18,23,24}$. Com a estruturação do serviço de Terapia Ocupacional na Ortopedia ficou definido 3 momentos que guiam o raciocínio clinico do TO: 1) Pré-operatório: foco está na manutenção das habilidades, orientações quanto a internação e precauções quanto o local com lesão para evitar desvio da fratura, embolia, dor e acidente; 2) Pós-operatório: foco está em treinar o usuário em tarefas essenciais para participação com segurança em domicilio; e 3) Tratamento conservador: foco está na prevenção de riscos e treino de tarefas com dispositivo de assistência recomendado (andador, muleta, elevador de perna, tipoia, dentre outros) garantido o alinhamento do segmento e a segurança do usuário ${ }^{15,16,21,22}$.

O estudo em questão foi submetido à aprovação do Comitê de Ética em Pesquisa da Universidade Federal de Minas Gerais sob registro CAAE: 17987713400005149 e cumpri os princípios éticos contidos na Declaração de Helsinki (2000). Por ser uma análise de prontuário retrospectivo e ser inviável a contactação de cada um dos pacientes a posteriori, não necessitou de Termo de Consentimento Livre e Esclarecido (TCLE). 


\section{RESULTADOS}

Em relação ao tempo de permanência no HRTN, dos 125 prontuários foi encontrada uma média de 16,33 dias $( \pm 18,09)$. No que se refere aos dados demográficos a Tabela 1 exibe os dados relevantes para o estudo.

Tabela 1 - Dados sociodemográficos

\begin{tabular}{lc}
\hline Variáveis & $\mathbf{N}=\mathbf{1 2 5}$ \\
\hline Gênero & \\
Feminino & $74,4 \%(93)$ \\
Masculino & $25,6 \%(32)$ \\
Idade (média \pm desvio padrão) em anos & 77,96 anos $( \pm 9,95)$ \\
Local residência & \\
Belo Horizonte & $73,6 \%(92)$ \\
Região Metropolitana & $26,4 \%(33)$ \\
\hline
\end{tabular}

Foi encontrado como diagnóstico mais frequente a fratura de fêmur com $68 \%$ ( 85 casos) dos casos, seguida pela fratura de membro superior $15,20 \%$ (19 casos). Os demais diagnósticos representam menos de $5 \%$ ( 1 a 7 casos) da amostra, são eles: fratura de tíbia, fratura de vértebras, luxação, fratura de joelho, atraso na consolidação de fratura, complicação de prótese de quadril e fratura de tornozelo.
Em relação às condutas ortopédicas destaca-se a osteossíntese de membros inferiores somando 49,6\% (62 casos), a artroplastia total de quadril (ATQ) $12,8 \%$ (16 casos), o tratamento conservador $12 \%$ (15 casos), artroplastia parcial do quadril (APQ) 7,2\% (9 casos) e osteossíntese de membros superiores $6,4 \%$ ( 8 casos). As demais condutas ortopédicas representam menos de 5\% (1 a 5 casos) da amostra, são elas: revisões de próteses, artrodese de coluna, retirada de haste intramedular de tíbia, reparo de tensão de joelho, redução cruenta e prótese e quadril, redução cruenta de luxação de quadril, pinça de confeiteiro, hemiatroplastia de úmero.

Foram encontrados 104 pacientes $(83,2 \%)$ que tiveram a MIF de admissão. Devido ao alto fluxo dos pacientes são frequentes internações breves, não sendo possível realizar a avaliação/reavaliação completa pela equipe da $\mathrm{TO}^{24}$. Em relação aos escores obtidos nestas MIF de admissão encontradas, a maior média está nas variáveis no domínio Cognitivo: Compreensão 5,09; Expressão 5,15; Interação social 5,60; Resolução problemas 4,66; e Memória 4,74. As piores médias referem-se às tarefas do domínio Motor, que envolvem principalmente a mobilidade de membros inferiores, como deambulação com média de 1,64, transferências com 1,80, banho com 1,84, vestir a metade inferior do corpo com 1,57.

No que se refere ao trabalho da equipe de TO, a Tabela 2 expõe um total de 729 atendimentos e 1963 intervenções realizadas com os 125 pacientes.

Tabela 2 - Intervenções em terapia ocupacional

\begin{tabular}{lcccc}
\hline Intervenção & Frequência & Porcentagem & Média & Desvio padrão \\
\hline Educativa & 519 & $26,4 \%$ & 4,15 & 4,32 \\
Função mental & 340 & $17,3 \%$ & 2,72 & 4,28 \\
Adaptativa & 323 & $16,4 \%$ & 2,58 & 4,79 \\
Mudar posição básica do corpo & 260 & $13,2 \%$ & 2,08 & 3,98 \\
Função neuromusulo-esquelética & 151 & $7,6 \%$ & 1,20 & 3,26 \\
Comer & 85 & $4,3 \%$ & 0,68 & 1,41 \\
Auto transferência & 73 & $3,7 \%$ & 0,58 & 1,19 \\
Manter posição básica do corpo & 68 & $3,4 \%$ & 0,54 & 1,41 \\
Vestir & 37 & $1,8 \%$ & 0,29 & 0,68 \\
Banho & 26 & $1,3 \%$ & 0,20 & 0,61 \\
Cuidar da pele & 18 & $0,91 \%$ & 0,14 & 0,75 \\
Despir & 15 & $0,76 \%$ & 0,12 & 0,41 \\
Protetora e reparadora da pele & 14 & $0,71 \%$ & 0,11 & 0,54 \\
Cuidar do cabelo & 12 & $0,61 \%$ & 0,09 & 0,38 \\
Cuidar dos dentes & 11 & $0,56 \%$ & 0,08 & 0,66 \\
Beber & 10 & $0,50 \%$ & 0,08 & 0,37 \\
Função sensorial & 1 & $0,05 \%$ & 0,008 & 0,08 \\
Total & 1963 & $100 \%$ & - & - \\
\hline
\end{tabular}


Na Tabela 3 analisou-se a proporção das intervenções mais realizadas pela Terapia Ocupacional de acordo com o tipo de conduta ortopédica. Foi analisado as intervenções mais frequentes por conduta ortopédica, por exemplo, $69 \%$ dos pacientes que passou pelo procedimento de ATQ recebeu pelo menos uma intervenção restauradora/da manutenção da função mental.
Na Tabela 4, destaca-se alto número de pacientes submetidos à osteossíntese de membros inferiores, ATQ e tratamento conservador. Por outro lado, redução cruenta de prótese de quadril e haste intramedular de tíbia representam apenas dois casos da amostra total, podendo ser considerados como casos atípicos.

Tabela 3 - Intervenções da Terapia Ocupacional mais frequentes por condutas ortopédicas

\begin{tabular}{|c|c|c|c|c|c|c|}
\hline $\begin{array}{c}\text { Invervenção } \\
\text { TO }\end{array}$ & $\begin{array}{l}\text { Osteo. } \\
\text { MMII }\end{array}$ & $\begin{array}{l}\text { Osteo. } \\
\text { MMSS }\end{array}$ & $\begin{array}{c}\text { TTO. } \\
\text { CONSERVADOR }\end{array}$ & ATQ & APQ & $\begin{array}{l}\text { Revisão de } \\
\text { prótese }\end{array}$ \\
\hline $1^{\circ}$ & $\begin{array}{c}\text { Educativa } \\
98 \%\end{array}$ & $\begin{array}{c}\text { Educativa } \\
100 \%\end{array}$ & $\begin{array}{c}\text { Educativa } \\
93 \%\end{array}$ & $\begin{array}{c}\text { Educativa } \\
100 \%\end{array}$ & $\begin{array}{c}\text { Educativa } \\
100 \%\end{array}$ & $\begin{array}{c}\text { Educativa } \\
100 \%\end{array}$ \\
\hline $2^{\circ}$ & $\begin{array}{c}\text { Mudar } \\
67 \%\end{array}$ & $\begin{array}{c}\text { Adaptativa } \\
75 \%\end{array}$ & $\begin{array}{c}\text { Adaptativa } \\
87 \%\end{array}$ & $\begin{array}{c}\text { Adaptativa } \\
81 \%\end{array}$ & $\begin{array}{c}\text { Adaptativa } \\
78 \%\end{array}$ & $\begin{array}{c}\text { Adaptativa } \\
100 \%\end{array}$ \\
\hline $3^{\circ}$ & $\begin{array}{l}\text { Mental } \\
62 \%\end{array}$ & $\begin{array}{c}\text { Neuromus } \\
37 \%\end{array}$ & $\begin{array}{c}\text { Mental } \\
53 \%\end{array}$ & $\begin{array}{c}\text { Mental } \\
69 \%\end{array}$ & $\begin{array}{c}\text { Mental } \\
78 \%\end{array}$ & $\begin{array}{c}\text { Mental } \\
100 \%\end{array}$ \\
\hline $4^{\circ}$ & $\begin{array}{c}\text { Adaptativa } \\
46 \%\end{array}$ & $\begin{array}{c}\text { Comer } \\
25 \%\end{array}$ & $\begin{array}{c}\text { Mudar } \\
47 \%\end{array}$ & $\begin{array}{c}\text { Mudar } \\
61 \%\end{array}$ & $\begin{array}{c}\text { Mudar } \\
78 \%\end{array}$ & $\begin{array}{c}\text { Banho } \\
100 \%\end{array}$ \\
\hline
\end{tabular}

Tabela 4 - Média de atendimentos da Terapia Ocupacional por diagnósticos clínicos por condutas ortopédicas

\begin{tabular}{|c|c|c|c|}
\hline Conduta Ortoédica & $\begin{array}{c}\text { Média de atendimentos } \\
\text { de TO } \\
\end{array}$ & Número de pacientes & $\begin{array}{c}\text { Média tempo de } \\
\text { permanência }\end{array}$ \\
\hline Redução cruenta de prótese de quadril & 33 & 1 & 70 \\
\hline Retirada de haste intramedular de tíbia & 18 & 1 & 65 \\
\hline Revisão de prótese & 17,2 & 5 & 45 \\
\hline Redução cruenta de luxação de quadril & 6 & 1 & 11 \\
\hline Artroplastia parcial do quadril & 5,7 & 9 & 14,6 \\
\hline Artoplastia total do quadril & 5,375 & 16 & 15 \\
\hline Osteossíntese de membros inferiores & 5,25 & 62 & 15,08 \\
\hline Tratamento conservador & 5,06 & 15 & 14,5 \\
\hline Artrodese coluna & 4,5 & 2 & 21 \\
\hline Osteossíntese de membros superiores & 3,5 & 8 & 8,25 \\
\hline Pinça de confeteiro & 3 & 1 & 3 \\
\hline Hemiatroplastia úmero & 2 & 1 & 14 \\
\hline
\end{tabular}

\section{DISCUSSÃO}

O tratamento do Terapeuta Ocupacional junto ao idoso com alterações ortopédicas é guiado pela importância em retomar a independência em tarefas específicas, necessárias para uma alta segura. Essas metas são frequentemente influenciadas pelo fluxo hospitalar intenso, que enfatiza a alta breve e taxas máximas de admissão. Neste ambiente, os terapeutas ocupacionais devem priorizar as tarefas mais importantes que o indivíduo precisa realizar para uma alta segura ${ }^{22}$.

A média de tempo de permanência hospitalar foi semelhante à encontrada na literatura de aproximadamente 15 dias, com alta variação $( \pm 18,09)^{10}$. Essa variação demonstra a heterogeneidade da amostra. Em relação aos tipos de fratura encontrados, a fratura de fêmur apresentou maior frequência o que também vai ao encontro da literatura ${ }^{13}$.

A literatura registra a maior incidência de fratura no gênero feminino. Nas mulheres a incidência é de duas a três vezes mais frequente que nos homens ${ }^{1,2,4,7,9}$. Os mecanismos para explicar esse fenômeno são pouco claros, sendo citados fatores como: maior perda de massa óssea, aumentando a probabilidade de osteoporose, maior prevalência de doenças crônicas, entre outros ${ }^{1}$.

Em relação aos escores encontrados na MIF de admissão, observamos pior pontuação nas tarefas que envolvem principalmente a mobilidade de membros 
inferiores, como transferências, banho, vestir a metade inferior do corpo, entre outros. Fato esperado, uma vez que no momento pré-operatório o paciente é orientado pela equipe de saúde sobre a importância de manter o seguimento recém fraturando o máximo imobilizado, impossibilitando a realização de diversas tarefas que envolvem a mobilidade. Além da presença de dor extrema no membro fraturado ao movimento, contribuindo para aumentar a imobilização deste seguimento.

A prática da Terapia Ocupacional com essa população em contexto hospitalar pode ser dividida em dois momentos, o pré-operatório e pós-operatório ${ }^{15,17,21,22}$. O primeiro com foco nas orientações quanto à internação e cuidados com o membro fraturado, na manutenção das habilidades e nas adaptações para melhorar o posicionamento do idoso no leito. $\mathrm{O}$ segundo momento é focado em treinar o usuário em tarefas essenciais, além de garantir aplicação das recomendações ortopédicas.

A intervenção educativa mais frequentemente encontrada neste estudo perpassa os dois momentos descritos, desde o momento da admissão até a alta, adquirindo ampla importância. Essa intervenção pode ser realizada por meio da orientação verbal e/ou entrega de cartilha ${ }^{15,16}$. Não é raro nas alterações ortopédicas o paciente necessitar de adaptar a maneira como realizam as atividades pós-operatórias, logo, estratégias compensatórias são comumente adotadas e a orientação, no contexto de uma abordagem educativa, é essencial para que essas estratégias sejam incorporadas às ocupações $\operatorname{cotidianas}^{21,22}$. Portanto a intervenção educativa realizada constantemente e é essencial para orientar o idoso e sua família sobre a nova condição de saúde, a forma como as tarefas devem ser realizadas, as precauções relacionadas ao posicionamento no leito, os movimentos que devem ser evitados de acordo com as recomendações ortopédicas durante as AVD, a importância do idoso permanecer o mais participativo nas AVD, além de orientações sobre os encaminhamentos entregues no momento da alta, promovendo a continuidade do cuidado na rede do Sistema Único de Saúde (SUS) $)^{15,16,17,22}$.

As intervenções restauradoras ou da manutenção da função mental - segunda mais frequente encontrada neste estudo - também perpassam os dois momentos da internação, uma vez que o paciente pode apresentar alguma alteração cognitiva prévia e/ou desenvolver delirium ao longo da internação. Alterações nas funções mentais prévias à internação, como as demências e as desencadeadas durante a internação hospitalar, como o delirium, impactam diretamente na capacidade funcional do idoso, influenciando negativamente o prognóstico funcional ${ }^{12}$. De acordo com a literatura, $30 \%$ dos idosos internados por questões ortopédicas desenvolvem delirium, o que pode estar relacionado ao quadro de dor e à imobilidade, entre outros $^{6,9,12}$. As alterações cognitivas neste idoso podem representar algo desafiador para o Terapeuta Ocupaciodenal, visto que para obter sucesso nas suas intervenções é fundamental que o idoso consiga compreender as estratégias ensinadas e incorporá-las no seu cotidiano ${ }^{22}$. Neste caso, estratégias compensatórias podem ser utilizadas como o uso de informações escritas, o reforço por parte de outros funcionários e o envolvimento da família ${ }^{22}$. A intervenção restauradora ou da manutenção da função mental também pode envolver a orientação espaço temporal, por meio de Terapia de Orientação para a Realidade (TOR), assim como estimulação de aspectos específicos da função mental, por meio do treino orientado para a tarefa ${ }^{22}$.

A terceira intervenção mais frequente encontrada neste estudo, intervenção adaptativa, também perpassa os dois momentos da internação. De acordo com a literatura, as lesões por pressão (escaras e úlceras) no paciente idoso internado representam uma complicação com graves consequências ${ }^{12,23}$. Portanto, o TO no momento pré-operatório tem como intervenção adaptativa principal confeccionar dispositivos de posicionamento, garantindo o alívio de pressão em porções do corpo que tem risco de lesão por pressão. Além deste risco, é de extrema importância o alinhamento do membro recém fraturado, portanto, o TO pode confeccionar dispositivos para melhor posicionar o membro evitando novos desvios na fratura ${ }^{21,22}$.

O diferencial da intervenção adaptativa no momento pós-operatório é a utilização de adaptações específicas para compensar funções facilitando o desempenho ocupacional do paciente $\mathrm{e}^{1,11,21,22}$. Essas adaptações são mais frequentes no paciente que foi submetido à protetização, uma vez que este deve seguir restrições de movimento de flexão de quadril, recomendadas pelo ortopedista. Logo, este paciente pode apresentar algumas limitações funcionais, sendo necessária a confecção de dispositivos como: elevador de perna útil nas transferências no leito, calçadeira adaptada e cabo alongado, para auxiliar na tarefa de vestir membros inferiores ${ }^{11,21,22}$.

A intervenção restauradora da atividade de mobilidade, quarta intervenção mais frequente, é a única intervenção das quatro aqui descritas que é realizada mais frequentemente no momento pós-operatório, uma vez que no momento pré-operatório o paciente não tolera mudanças de posição do corpo (sentar-se, levantar-se, mudar de decúbito ativamente, entre outros). Esta intervenção consiste em treinar o paciente a utilizar estratégias compensatórias durante as tarefas de mobilidade, como: 
transferências utilizando apoio unilateral e transferências no leito utilizando o elevador de perna ou a escada de cama, dentre outros ${ }^{11,16}$

Estudo sobre a prática da TO nas precauções pós ATQ encontrou como principais atividades treinadas pelos TOs, as tarefas que envolvem mobilidade, como: transferências no leito, transferências entre cadeiras e transferências no banheiro ${ }^{22}$. O treino de atividades que envolvem mobilidade ganha maior relevância com pacientes protetizados, devido às precauções que devem ser tomadas durante as transferências, por aproximadamente três meses após a cirurgia ${ }^{21,22} \mathrm{Os}$ movimentos que uma pessoa é aconselhada a evitar após a realização de uma ATQ são geralmente, flexão de quadril além de $90^{\circ}$, adução na linha média e rotação externa e interna ${ }^{22}$.É papel do terapeuta ocupacional ensinar estratégias e resolver problemas com o idoso durante a realização das tarefas para garantir que as precauções sejam aplicadas às ocupações específicas do idoso, justificando a importância do foco da Terapia Ocupacional neste estudo com nas intervenções que envolvem mobilidade com esse paciente ${ }^{22}$.

Nos pacientes submetidos à osteossíntese de membros superiores, a intervenção restauradora ou da manutenção da função neuromusculoesquelética, assim como o treino funcional da atividade comer foram intervenções diferenciais. A grande maioria dos pacientes desenvolve edema em todo o seguimento afetado, mais agudo nas mãos, sendo a intervenção restauradora ou da manutenção da função neuromusculoesquelética de extrema importância nesse tipo de lesão, uma vez que a mobilização ativa é a principal intervenção para a redução do edema local, prevenindo complicações como rigidez articular, edema crônico, dor e claro perda de função manual ${ }^{24}$. A rápida perda ou redução da função manual devido à imobilização do seguimento fraturado impacta negativamente na independência do paciente, sendo necessário o treino funcional de tarefas bimanuais, como a alimentação, para aprendizado de novas estratégias unimanuais, como métodos compensatórios para neutralizar a incapacidade e/ou a influência do ambiente ${ }^{11,22}$.

Identifica-se a revisão de prótese, como o procedimento de maior média de atendimentos de TO, com alto tempo de permanência hospitalar e número considerável de pacientes.

De acordo com a literatura, o paciente que passa pela revisão de prótese de quadril necessita de atenção específica de toda a equipe, uma vez que necessitará seguir rigorosas precauções pós-procedimento, para evitar novas complicações. Logo, a atuação da TO junto a esses pacientes tem como principal objetivo orientá-los sobre as principais recomendações, e treiná-los a executar suas AVD com a nova limitação de movimento ${ }^{16}$. Acredita-se que isso explica o fato do treino funcional de banho aparecer, neste estudo, como uma das principais intervenções realizadas pela TO com os pacientes submetidos a revisão de prótese, sendo uma atividade que engloba mobilidade de quadril, sendo extremamente importante que o TO ensine ao paciente estratégias compensatórias que protejam a articulação do quadril, para que ele possa desempenhar essa tarefa de forma independente.

\section{CONCLUSÃO}

Com o envelhecimento populacional observado nos últimos anos, os profissionais da saúde têm uma população extensa de idosos frágeis com alterações de saúde, muitas das vezes, resultante de uma somatória de comorbidade. As alterações ortopédicas, como as fraturas, se encaixam neste perfil e tem gerado a hospitalização destes idosos que podem ter sua capacidade funcional diminuída.

Assim, a Terapia Ocupacional tem papel fundamental junto à este idoso e sua família, tendo como benefícios gerais do atendimento o treinamento de atividades de vida diária para aprendizagem de estratégias compensatórias, manejo e prevenção da dor, prevenção de quedas, proteção da região da fratura e uso de dispositivos de assistência. Frente ao momento agudo de internação hospitalar, momento esse propício a complicações adicionais foi observado que o TO apresenta como foco principal de suas intervenções a prevenção e/ou tratamento de possíveis complicações como, dor, lesões por pressão, delirium, edema, entre outros. Tais complicações podem aumentar o tempo de permanência hospitalar. Além deste foco primário foi observado que é parte da prática da Terapia Ocupacional junto ao idoso com alterações ortopédicas, promover a participação do idoso nas atividades funcionais possíveis ainda durante a internação hospitalar, para que ele seja capaz de retomar seus papéis ocupacionais em domicílio.

Participação dos autores: Helena Auler Loureiro: Autora principal, residente alocada no cenário onde foi realizado o estudo. Responsável pela concepção e delineamento dos dados bem como redação e interpretação dos dados do estudo. Kênia Lara Silva: Co-autora e orientadora da residente em questão. Participou do delineamento e interpretação dos dados com crítica intelectual importante para o conteúdo final. Marcela Aline Fernandes Braga: Co-autora e Co-orientadora da residente. Participou da concepção, análise e interpretação dos dados. Auxiliou na redação do manuscrito bem como suas constantes revisões. 


\section{REFERÊNCIAS}

1. Cruz DT, Ribeiro LC, Vieira MT, Teixeira MTB, Bastos RR, Leite ICG. Prevalência de quedas e fatores associados em idosos. Rev Saúde Pública. 2012;46(1):138-46. http://dx.doi. org/10.1590/S0034-89102011005000087.

2. Martini FP, Guerra MTE, Mendes MR, Echeveste SS. Estudo epidemiológico das fraturas de fềmur proximal em Canoas no ano de 2010. Rev AMRIGS. 2012;56(4):320-4.

3. Minayo MCS. O envelhecimento da população brasileira e os desafios para o setor saúde. Cad Saúde Pública. 2012;28(2):208-9.

4. Montalbán-Quesada S, Garcia-Garcia I, Moreno-Lorenzo C. Functional evolution in elderly individuals with hip fracture surgery. Rev Esc Enferm USP. 2012;46(5):1095-1100. http:// dx.doi.org/10.1590/S0080-62342012000500009.

5. Couto MAS, Reiff R, Castro AP. Funcionalidade após a cirurgia de quadril: correlação entre equilíbrio, idade, independência e depressão em idosos. Acta Fisiatr. 2012;19(1):32-6. doi: 10.5935/0104-7795.20120007.

6. Hung WW, Egol KA, Zuckerman JD, Siu AL. Hip fracture management tailoring care for the older patient. JAMA. 2012;307(20):2185-94. doi:10.1001/jama.2012.4842.

7. Ricci G, Longaray MP, Gonçalves RZ, Ungaretti Neto AS, Manente M, Barbosa LBH. Avaliação da taxa de mortalidade em um ano após fratura do quadril e fatores relacionados à diminuição de sobrevida no idoso. Rev Bras Ortop. 2012;47(3):304-9. https:// dx.doi.org/10.1590/S0102-36162012000300005.

8. Karni S, Bentur N, Ratzon N. Participation and quality of life of cognitively impaired older women in Israel following hip fractures. Occup Ther Int. 2014;21(3):91-7. https://doi. org/10.1002/oti.1365.

9. Close JD, Swartz K, Deu R. Hip fracture in older patients: tips and tools to speed recovery. J Fam Pract. 2013;62(9):484-92.

10. Saltvedt I, Prestmo A, Einarsen E, Johnsen LG, Helbostad JL, Sletvold O. Development and delivery of patient treatment in the Trondheim Hip Fracture trial. A new geriatric in-hospital pathway for elderly patients with hip fracture. BMC Res Notes. 2012;5:355. doi: 10.1186/1756-0500-5-355.

11. Pierce SL. Restaurando a mobilidade. In: Trombly CA, Radomski MV. Terapia ocupacional para disfunções físicas. São Paulo: Santos; 2013. p.817-53.

12. Leme LEG, Sitta MC, Toledo M. Cirurgia ortopédica em idosos: aspectos clínicos. Rev Bras Ortop. 2011;46(3):238-46. doi: 10.1590/s0102-36162011000300002.

13. Del Duca GF, Antes DL, HallalII PC. Quedas e fraturas entre residentes de instituições de longa permanência para idosos.
Rev Bras Epidemiol. 2013;16(1):68-76. https://doi.org/10.1590/ S1415-790X2013000100007.

14. World Health Organization (WHO). International Classification of Functioning, Disability and Health: ICF. Geneve: World Health Organization; 2012. Available from: https://www.who. int/classifications/icf/en/.

15. Maher C, Bear-Lehman J. Condições ortopédicas. In: Trombly CA, Radomski MV. Terapia ocupacional para disfunções físicas. São Paulo: Santos; 2013. p.1106-30.

16. Coupe J, Gibb C, Harrison S, McCourt S, Porter L, Robertson K. Occupational therapy for adults undergoing total hip replacement: practice guideline. London: College of Occupational Therapists; 2012. Available from: https://www.rcot.co.uk/sites/default/files/ Total-Hip-Replacement.pdf.

17. Moores A. Occupational therapy following elective lower limb surgery In: Mooney M, Ireson C. Occupational Therapy in Orthopaedics and Trauma. United Kindon: John Wiley \& Sons Ltd; 2012. p.29-46.

18. Guimarães FAM, Lima RR, Souza AC, Livani B, Belangero WD. Avaliação da qualidade de vida em pacientes idosos um ano após o tratamento cirúrgico de fraturas transtrocanterianas do fềmur. Rev Bras Ortop. 2011;46(Suppl 1):48-54. https://doi. org/10.1590/S0102-36162011000700012.

19. Brucki SMD, Nitrini R, Caramelli P, Bertolucci PHF, Okamoto IH. Sugestões para o uso do Mini-Exame do Estado Mental no Brasil. Arq Neuropsiquiatr. 2003;61(3-B):777-81. doi: 10.1590/ s0004-282x2003000500014.

20. Riberto M, Miyasaki MH, Jucá SSH, Sakamoto H, Potiguara P, Pinto N. Validação da versão brasileira da Medida de Independência Funcional. Acta Fisiátr. 2004;11(2):72-6. https:// orcid.org/0000-0001-9549-8830.

21. Hoppenfeld S, Murthy VL. Tratamento e reabilitação de fraturas. São Paulo: Manole; 2001.

22. Mooney M, Ireson C. Occupational therapy in orthopaedics and trauma. Hong Kong: John Wiley \& Sons Ltd.; 2009.

23. Rogenski NMB, Kurcgant P. Incidência de úlceras por pressão após a implementação de um protocolo de prevenção. Rev Latino-Am Enfermagem. 2012;20(2):333-9. doi: https://doi. org/10.1590/S0104-11692012000200016.

24. Clé PGV, Tasso LE, Barbosa IR, Fonseca MCR, Elui VMC, Roncaglia FB, Mazzer N, Barbieri CH. Estudo retrospectivo do estado funcional de pacientes com fratura do rádio distal submetidos à osteossíntese com placa LCP. Acta Fisiatr. 2011;18 (4):163-8. http://dx.doi.org/10.5935/0104-7795.20110001. 